\title{
Enhanced Automated and Secure Parking Structure \& Tactics
}

\author{
Vikrant Chatole $^{1}$, Hitesh Pogade ${ }^{2}$, Aniket Mahajan ${ }^{3}$, Er. Chandan Prasad ${ }^{4}$ \\ B. Tech Scholar, CTIS, School of Information \& Technology, Pune, India ${ }^{1}$ \\ BCA Scholar, CTIS, School of Information \& Technology, Pune, India ${ }^{2}$ \\ BCA Scholar, MACT, School of Information \& Technology, Pune, India ${ }^{3}$ \\ Assistant Professor, IT, Ajeenkya DY Patil University, Pune, India ${ }^{4}$
}

\begin{abstract}
The swift improvement in Economic, Social and technological aspects has taken lifestyle to another level. The level at which the current management systems lag behind or barely keep up with the ever-changing and ever evolving standards of living. Apart from that, an even bigger threat is the immensely growing populace and of course, their Vehicles. The Paper discusses the complications of a major part of Vehicle management, The Parking. Providing a solution for the hurdles faced in the running Parking Systems using a complete automation of every manual operation with an integrated computing system to overcome the hurdle and further more to improvise the management.
\end{abstract}

Keywords: Smart Parking, Parking Management, Pressure Sensors, RFID-Radio Frequency Identifier, Vehicle Theft.

\section{INTRODUCTION}

As a side of urbanization and overpopulation, the vehicles has raised at extremes. The increased number of vehicles has directly resulted in the long queues and time taking processes involving security issues. These are the processes which are time taking though can't be compromised on. If skipped, we surely are putting our vehicle at a high risk since the current systems require no personal verification for the return of the vehicle and loosely bounded on the manual workers to look after. The human labour and in-charge people/authority already handles more than a hundred vehicles and people owning them per hour and unfortunately are incapable of assuring a full roof security to every individual. With a little planning and presence of mind, one can easily plunder or even take possession of the vehicle and drive their way out without even being recognised as Vehicle Theft until it's too late.

These Huge Parking lots are no less than mazes and can be very confusing, time taking and puzzling at times to find one's own vehicle.

The problems originated from human intervened control systems can be all be rectified by implementing a total automation of the system. A totally Computerised and fully automated system that works under certain protocols leaving no space for human error and can not only allocate and deallocate a parking space for a vehicle but also Look after the vehicle when parked, Act as a small data centre of vehicles for further use of information later in future, (for applications like crime investigation). In other words, a management system that's not only good at parking vehicles but also helpful in investigating crimes like Vehicle Theft, Illegal Possession of the Vehicle and even more.

\section{RELATED WORK}

Several works and Innovations in the field of vehicle parking management has been carried out. Reference [1] is an IoT based system depending on RFID sensors and lets the user pre-book a parking spot and involves a virtual smart card to pay the bills but limits at pre-timed scheduled entries [2] is an IoT based mechanism to allot a parking spot to the user but only takes in the minimum driving distance as deciding parameter. Checks[3] in and check out using RFID but fails to provide the user the freedom to choose a parking spot on his/her own will. Apart from that it also fails to generate an automated and computerised bill.This is [4] an automated system to open and close the door and trigger a buzzer if the driver is drunk but doesn't consider automated bill generation [5] is a system to record and look after the number of free parking slot available and permit vehicles accordingly. In this[6] an Infra-Red sensor depended concept of Automatic Multilevel Car Parking, to reduce find time consumed in finding an empty parking slot. Both [5] and [6] fail to generate a computerised bill

\section{III.PROPOSED SYSTEM}

The system incorporates embedded devices and wireless information transfer to communicate and operate. The system depends on RFID Tags as verification and 


\section{ISO 3297:2007 Certified}

Vol. 5, Issue 3, March 2017

information input parameter. The proposed system is consist of two models:

- Entrance Model

- Departure/Exit Model

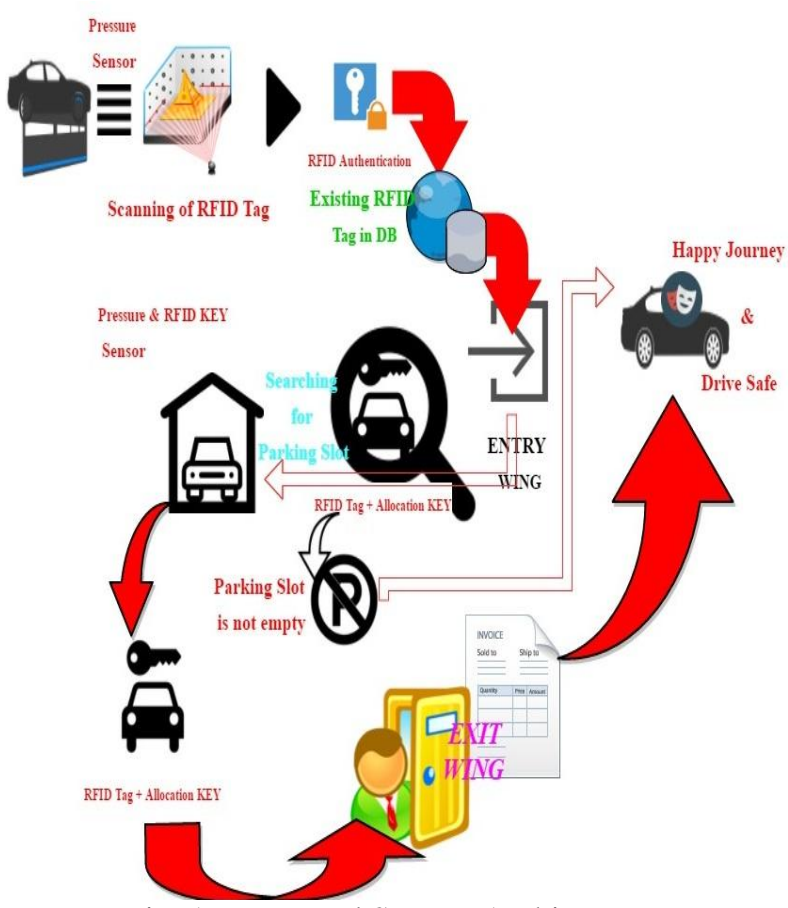

Fig. 1. Proposed System Architecture

\section{A. Entrance Model}

At the Entrance Gate, Pressure sensors are placed to recognise a vehicle arrival. Soon as the Pressure sensors start responding assertively, the turns the scanner on to scan the RFID tag of the incoming vehicle. As the RFID information is gained through the scan.

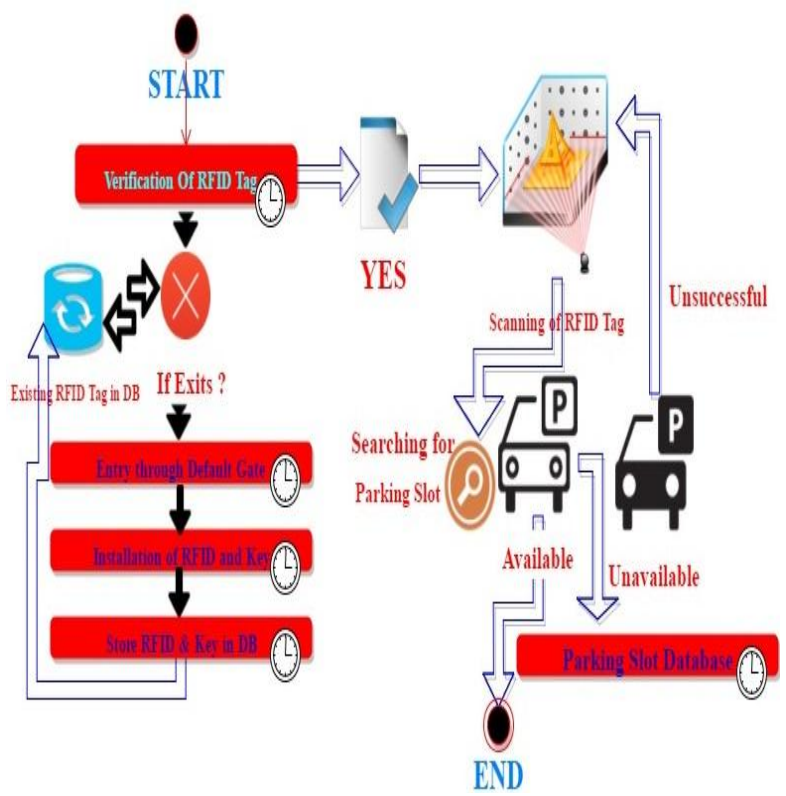

Fig. 2. Flow of Entrance Model
The information is then looked in the current existing database of the system, if any matches are discovered and are verified by the system database, the system starts the search for an empty parking slot. If no matches are found in the database prior then the user is granted an entry through the default gate which executes installation of RFID tag and RFID tag key. The respective RFID Tag and RFID key are then profiled in the database and the parking slot is then checked for the respective Vehicle.

To find an empty parking slot the system looks for a slots with their pressure sensors returning null values i.e. no pressure/weight which implies absence of vehicle, this way the database regulates the engaged and free parking count. If a free parking slot is found, it is assigned to the user. As the Vehicle is now granted a Parking spot and the vehicle is parked the Electronic gates locks and the Parking Process completes.

\section{B. Departure/Exit Model}

The Departure process involves pretty simple and fast procedures considering a user might need to rush and cannot afford a whole long process to be done and yet without compromising the security. The Parked Vehicle stays locked into the assigned parking spot until the user tries to drive his/her way out. At every electronic gate a RFID scanner is placed which on exit, communicate with the RFID tag. If the RFID key tag has been successfully scanned and Verified by the database the Gate will be unlocked to leave OR if the RFID Tag scanning and verification process goes through any complications such as unrecognizability, Damaged RFID tag or absence of RFID Tag, the authority will be immediately alarmed in the control room about the malfunction, since it potentially can be a case of vehicle theft/robbery.

The Authority can then act accordingly. With an automated bill generated and being paid by the user the vehicle is free to leave. As the Vehicle Exits, the checkout time of the vehicle is then added to the database file and the parking spot is again counted as unengaged by the database and is free to use again. The cycle of the allocation and deallocation for a parking spot for a vehicle hence completes here.

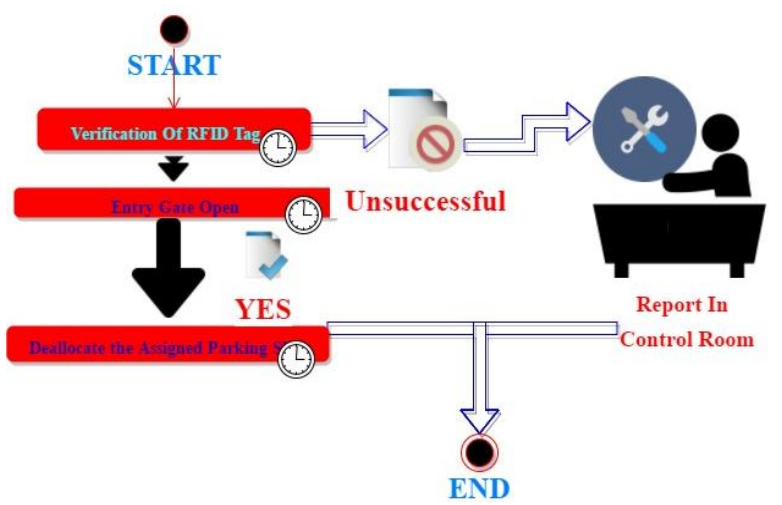

Fig. 3. Flow of Departure / Exit Model 
Vol. 5, Issue 3, March 2017

\section{IV.FUTURE SCOPES AND CONCLUSION}

The Proposed system, in implementation can not only reduce but cut the hassles of a safe vehicle parking multiple fold. The System completely rectifies any possible human error by complete automation and computerized mechanisms. Right from entering the Parking Gate to Departing. Apart from that, it also grants the user and authority to transact money hurdle freely by auto generated bills. The "ENHANCED AUTOMATED AND SECURE PARKING STRUCTURE \& TACTIC" enhances and advances the current Parking systems with swift speed improvements and additional security and makes sure that a driver doesn't make a bad memory about their vehicle in the parking premise.

\section{REFERENCES}

[1] Vatsala Daur, Prerit Bhandari, Laxay Jain, Nalini N, "Smart Car Parking System", International Journal of Advanced Research in Computer Science and Software Engineering Volume 6, Issue 5, May 2016

[2] V. Hans, , P. S. Sethi, , J. Kinra, "An approach to IoT based car parking and reservation system on Cloud", 2015 International Conference on Green Computing and Internet of Things (ICGCIoT). 2015

[3] P. Parkhi, S. Thakur and S. Chauhan, "RFID-based Parking Management System", International Journal of Advanced Research in Computer and Communication Engineering, vol. 3, no. 2, 2014.

[4] Supriya Sunil Kadam, Monali Manoj Desai, Priyanka Ganpati Deshmukh and Vijaymala Sadashiv Shinde, "RFID Based Car Parking Security System Using Microcontroller IC89c52”, IJERT, vol. 4, no. 03, 2015.

[5] E. Soni, K. Kaur and A. Kumar, "Design And Development Of RFID Based Automated Car Parking System", The International Journal of Mathematics, Science, Technology and Management, vol. 2 , no. 2, p. 3, 2016

[6] A. Gupta, A. Jaiswar, H. Agarwal and C. Shankar, "Automatic Multilevel Car Parking", International Journal of Electrical and Electronics Research, vol. 3, no. 2, p. 4, 2015. 